\title{
A New Possibility of Dynamical Study on Solid State lonic Materials by Inelastic Neutron Scattering
}

\author{
M. Nakamura ${ }^{{ }^{\star}}$, K. Nakajima1 ${ }^{1}$, Y. Inamura ${ }^{1}$, S. Ohira-Kawamura ${ }^{1}$, T. Kikuchi ${ }^{1}$, \\ T. Otomo ${ }^{1,2}$ and M. Arai ${ }^{1}$ \\ ${ }^{1}$ Materials and Life Science Division, J-PARC Center, Tokai, Ibaraki, 319-1195, Japan \\ ${ }^{2}$ Institute of Materials Structure Science, High energy Accelerator Research Organization (KEK), Tsukuba, Ibaraki 305-0801, Japan
}

\author{
ARTICLE INFO \\ Article history: \\ Received 30 September 2010 \\ Received in Revised form 25 October 2010 \\ Accepted 26 October 2010 \\ Keywords: \\ Inelastic neutron scattering \\ J-PARC \\ Pulsed neutron source \\ Pulsed neutron techniques \\ Solid state ionic materials
}

\begin{abstract}
A B S T R A C T
A new technique of inelastic neutron scattering measurement utilizing the multiple incident energies is applied to the dynamical study of vitreous silica. A wide variety of extracted information from a series of two-dimensional maps of dynamical structure factor with multiple different incident energies are greatly valuable. The applicability and its expected contribution of new experimental technique into the further progress of scientific activities in solid state ionic materials are discussed.
\end{abstract}

(C) 2010 Atom Indonesia. All rights reserved

\section{INTRODUCTION}

Neutron scattering measurements have played an important role in the study of solid state ionic materials. For example, diffraction measurement provided a lot of structural information, and also quasi-elastic neutron scattering (QENS) measurement revealed the diffusion mechanism of mobile ions. On the other hand, inelastic neutron scattering (INS) measurement was not a major experimental method in the dynamical study of solid state ionic materials due to the low flux of neutron beam. However, the MW-class pulsed neutron source such a Japan Proton Accelerator Research Complex (J-PARC) may completely change the situation. The INS measurement with pulsed neutron source is able to provide a new stage for the dynamical study of solid state ionic materials through a two-dimensional map of dynamical structure factor $S(Q, E)$ over wide $Q-E$ range. We can easily extract a series of constant $Q$ or $E$ slices and also the integration over the arbitrary $Q$ or $E$ ranges from $S(Q, E)$ map. In fact, our previous works on superionic conductors using INS measurement with pulsed neutron source have given new insight into the origin of ionic conductivity in the solid state [1-4]. As an example, the comparison of low-energy dynamics between superionic conducting glass $(\mathrm{AgI})_{0.5}\left(\mathrm{AgPO}_{3}\right)_{0.5}$ and insulator glass $\mathrm{AgPO}_{3}$ was

\footnotetext{
* Corresponding author.

E-mail address:. mitsutaka.nakamura@j-parc.jp ( M. Nakamura )
}

illustrated in Fig. 1. The low-energy intensity (below $5 \mathrm{meV}$ ) of superionic conducting glass is much larger than that of insulator glass as shown in Fig. 1(a). One of the greatest benefits of INS measurement is that we can discuss the $Q$-dependent features of inelastic signal. Figure 1(b) shows the constant- $E$ slices $(1 \mathrm{meV}<E<3 \mathrm{meV})$ for both $(\mathrm{AgI})_{0.5}\left(\mathrm{AgPO}_{3}\right)_{0.5}$ and $\mathrm{AgPO}_{3}$, which clearly indicates that an excess low-energy intensity of superionic conducting glass is caused by a unique dynamics in the $Q$ range beyond around $2 \AA^{-1}$.

Furthermore, we have succeeded in experimentally demonstrating that a series of two-dimensional maps of $S(Q, E)$ with multiple incident energies can be simultaneously obtained by one measurement (multi-Ei measurement). The first demonstration of multi-Ei measurement was conducted on the observation of magnetic excitations in the spin-Peierls compound $\mathrm{CuGeO}_{3}$ single crystals using a Fermi chopper spectrometer 4SEASONS at J-PARC [5]. The multi-Ei measurement reduces the dead time of time-of-flight (TOF) measurement, and thus, it markedly increases the INS measurement efficiency [6]. Thus a new experimental technique is also markedly powerful for the dynamical study of isotropic materials, such as glass, liquid, and polycrystalline materials, which are typical form of solid state ionic materials. Accordingly, we are now ready for the breakthrough of scientific activities on the solid state ionic materials by making full use of both high neutron 
flux realized at J-PARC and novel experimental technique. In this study, multi-Ei measurement is applied to the dynamical study of vitreous silica $\left(v-\mathrm{SiO}_{2}\right)$ which is the most famous isotropic material, and the availability of multi-Ei measurement in the dynamical study of solid state ionic materials is validated.
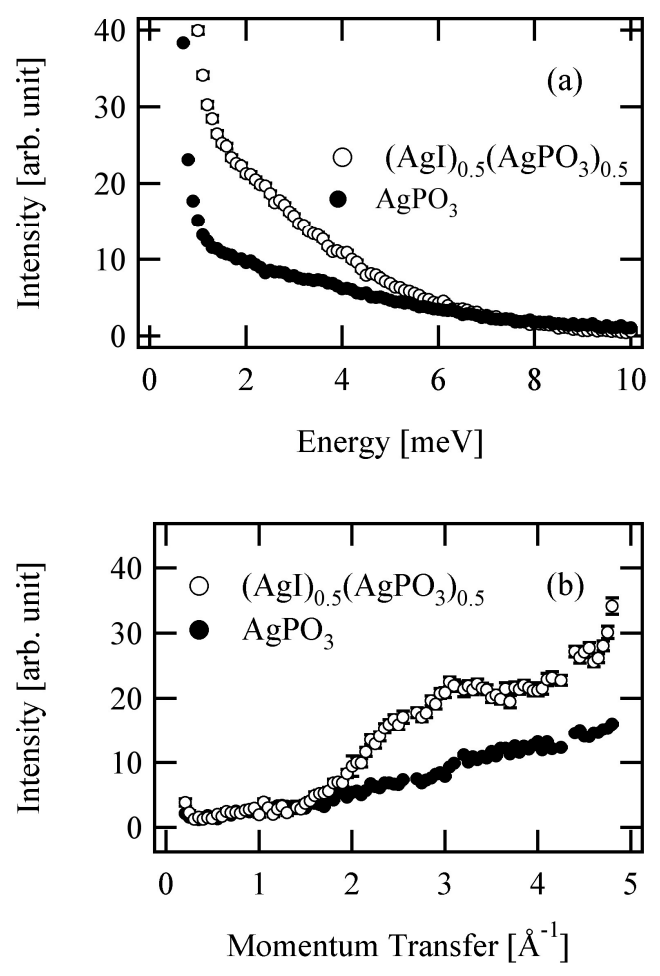

Fig. 1. Comparison of inelastic scattering between superionic conducting glass $(\mathrm{AgI})_{0.5}\left(\mathrm{AgPO}_{3}\right)_{0.5}$ and insulator glass $\mathrm{AgPO}_{3}$ [1]. All data were measured by MARI spectrometer at the ISIS facility, UK, and the environmental temperature was room temperature; (a) the energy dependence of $S(Q, E)$ obtained by the total integration along the $Q$ direction, and (b) the constant$E$ slices of $S(Q, E)$ integrated between $1 \mathrm{meV}$ and $3 \mathrm{meV}$.

\section{EXPERIMENTAL METHODS}

The multi-Ei measurement of $v-\mathrm{SiO}_{2}$ was carried out on the AMATERAS spectrometer at the J-PARC. AMATERAS is a direct geometry diskchopper spectrometer and designed for wide momentum-energy space measurement with high resolution and high flux [7]. The incident beam flux and resolutions of AMATERAS are flexibly tuned by changing the parameters of multiple disk choppers, such as the rotation speed and slit size. The moderator-sample distance $\left(L_{1}\right)$, the sample - detector distance $\left(L_{2}\right)$, and the monochromating chopper-sample distance $\left(L_{3}\right)$ are $30,4,1.6 \mathrm{~m}$, respectively. $\varphi 25.4 \mathrm{~mm} \times 2.9 \mathrm{~m}$ position-sensitive detectors (PSDs) are cylindrically arranged with a radius of $L_{2}$ around the sample. The data are collected using a newly developed eventmode data acquisition system, where each neutron captured by PSD is tagged with time stamp and stored over the source period ( $40 \mathrm{~ms}$ in J-PARC) event by event [8.9]. This data acquisition system enables users to freely pick up and reedit the TOF data sets based on the tag information. Several TOF data sets are independently analyzed for each inelastic scattering process in multi-Ei measurement.

Incident energy is arbitrarily selected by tuning the phase delay of monochromating chopper. Usual monochromating chopper frequency $(100-600 \mathrm{~Hz})$ is greatly larger than the accelerator frequency of pulsed neutron source $(25 \mathrm{~Hz}$ in J-PARC). It follows that chopper window is opening many times with in 1-frame of accelerator period (40 ms in J-PARC). Accordingly, multiple incident energy neutrons can pass through a monocromating chopper. A general formula of the $n$th incident wavelength $\lambda_{i}(\mathrm{n})[\AA]$ selected by a monochromating chopper [6] is

$$
\lambda_{i}(n)=\lambda_{i}(1)+\frac{3956(n-1)}{\left(L_{1}-L_{3}\right) f},
$$

where $\lambda_{i}(1)[\AA]$ is the shortest wavelength among the multiple incident neutrons, $f[\mathrm{~Hz}]$ the frequency of monochromating chopper. The unit of $L_{1}$ and $L_{3}$ in Equation (1) is meter. Incident energy $E_{i}[\mathrm{meV}]$ is calculated by a following relation with incident wavelength $\lambda_{i}[\AA]$

$$
E_{i}=\frac{81.81}{\lambda_{i}^{2}} .
$$

In this experiment, monochromating chopper frequency is $150 \mathrm{~Hz}$, and selected incident energies are $94.1,23.6,10.5$, and $5.9 \mathrm{meV}$. The incident beam size was $20 \mathrm{~mm}$ (width) $\times 30 \mathrm{~mm}$ (height). The sample of $v-\mathrm{SiO}_{2}$ was columnar form. Its diameter was $9 \mathrm{~mm}$ and its length was $80 \mathrm{~mm}$. The measurement was carried out at room temperature.

\section{RESULTS AND DISCUSSION}

Figure 2 shows the TOF spectrum of $v-\mathrm{SiO}_{2}$, which accumulate the data from all the detector pixels in the horizontal plane. We can observe several elastic peaks at every $\sim 7980 \mu \mathrm{s}$, which corresponds to the rotation period of the monochromating chopper at the detector position, i.e., $1 / f[\mathrm{~Hz}] \times\left(L_{1}+L_{2}\right) /\left(L_{1}-L_{3}\right) \times 10^{6} \mu$ s. These peaks correspond to $E_{i}=94.1,23.6,10.5$, and $5.9 \mathrm{meV}$ in order of arrival time. Inelastic processes 
for multiple $E_{i}$ 's are independently analyzed, and thus, we obtained four different $S(Q, E)$ maps from one data set as shown in Fig. 3. One can see an overall feature of the excitations from the data with high $E_{i}$, while the data with small $E_{i}$ gives precise information at low-energy region. In other words, the $S(Q, E)$ map with high $E_{i}$ and that with low $E_{i}$ have a zoom-in/out relation each other. Instrumental noise and background brought about several streaks at $E \sim 8 \mathrm{meV}$ in Fig. 3(c) and $E \sim 2 \mathrm{meV}$ in Fig. 3(d), where now we have already succeeded to suppress these fake signals.

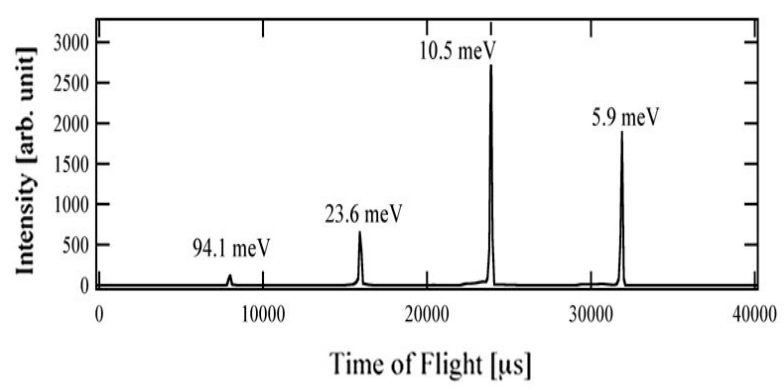

Fig. 2. TOF spectrum of $v-\mathrm{SiO}_{2}$. Data were collected using detector pixels positioned in the horizontal plane. The monochromating chopper frequency was $150 \mathrm{~Hz}$, and phase delay was tuned to the incident energy of $23.6 \mathrm{meV}$. Other incident neutrons with different energies were also concomitantly delivered to the sample.

Until now, enormous works have been done on both the structure and the dynamical properties of $v-\mathrm{SiO}_{2}$, which have been the subject of many review articles (for example, see [10]). A lot of valuable information can be extracted from the respective $S(Q, E)$ maps shown in Fig. 3. Some results are given in Fig. 4, and thus, we can examine the quality of new obtained data as compared with the results published in the literature. Fig. 4(a) shows the constant- $E$ slices of $S(Q, E)$ map with $E_{i}=94.1 \mathrm{meV}$, where the selected energy transfers are $15,30,45,60 \mathrm{meV}$. Each intensity is subtracted by constant background and then divided by $Q^{2}$. It is obvious that there exists the dispersive behavior up to $60 \mathrm{meV}$ despite the lack of structural periodicity. This result is consistent with previous works $[11,12]$. The $E$ dependence of $S(Q, E)$ with $E_{i}=23.6$ $\mathrm{meV}$, obtained by the total integration over $Q$, is plotted in Fig. 4(b). The so-called Boson Peak is clearly observed. More detailed investigations on the $Q$ dependence of Boson Peak is also feasible from the data with middle $E_{i}$ 's, such as $E_{i}=23.6$ or $10.5 \mathrm{meV}$. Elastic parts of $S(Q, E)$ with both $E_{i}=94.1$ $\mathrm{meV}$ and $E_{i}=5.9 \mathrm{meV}$ are shown in Fig. 4(c) and Fig. 4(d), respectively. For both spectra, the first sharp diffraction peak (FSDP) is observed around $1.5 \AA^{-1}$. Fig. 4(c) gives us the spectral features over wide $Q$ range, while Fig. 4(d) represents finer spectral features in the limited $Q$ region. The appearance of a weak shoulder below $1.0 \AA^{-1}$ can be seen in Fig. 4(d).

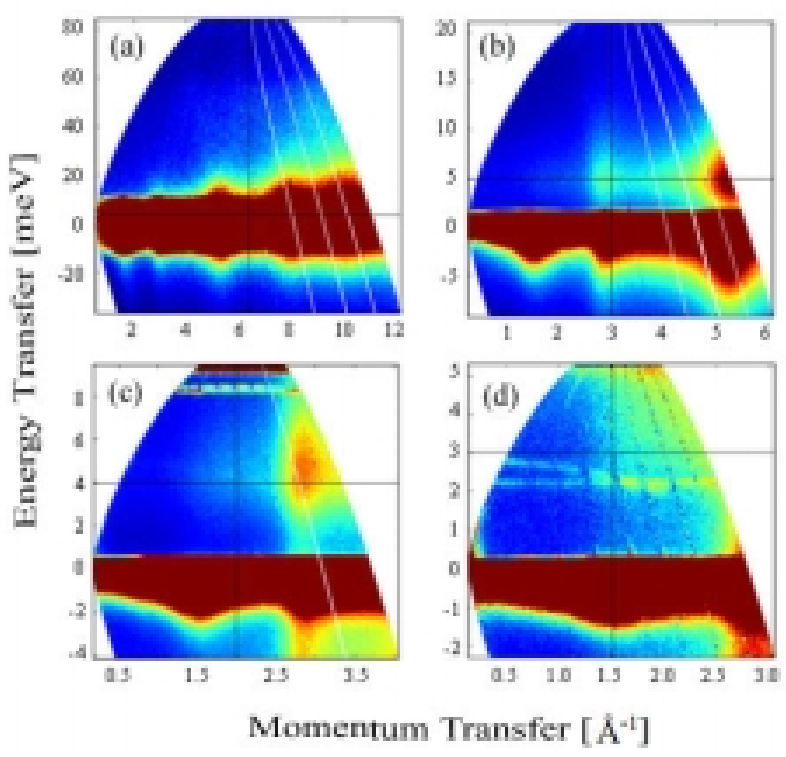

Fig. 3. Simultaneous observation of multiple two-dimensional $S(Q, E)$ maps for $v$ - $\mathrm{SiO}_{2}$ by one measurement. The horizontal axis shows the momentum transfer and the vertical axis shows the energy transfer. The incident energies are (a) 94.1, (b) 23.6, (c) 10.5 , and (d) $5.9 \mathrm{meV}$.

Next, let us discuss the availability of multiEi measurement for the study of solid state ionic materials. The data with high $E_{i}$ covers the wide $Q$ range, from which we can estimate the Debye-Waller factor and averaged mean-square displacement [13]. The temperature dependence or composition dependence of averaged mean-square displacement should yield valuable information on the mechanism of ionic conductivity. In addition, higher incident energy measurement more than $100 \mathrm{meV}$ may indicate some dynamical changes in connection with the activation energy of mobile ions which is usually in the range between $0.1 \mathrm{eV}$ and $1 \mathrm{eV}$. On the other hand, the data with small $E_{i}$ provides more detailed information in the lower $Q$ range. Detailed investigation on the $Q$ dependence of low-energy excitation in the solid state ionic materials will promote better understanding of peculiar dynamical properties presented by the previous experiments at the ISIS [1-4]. It is well known that the structure factor of superionic conducting glass exhibits a strong and relatively sharp diffraction peak at anomalously low values of $Q\left(0.6<Q<0.8 \AA^{-1}\right)[14,15]$. The data with smaller $E_{i}(<10 \mathrm{meV})$ will enable to develop the dynamical study of this anomalous prepeak. 

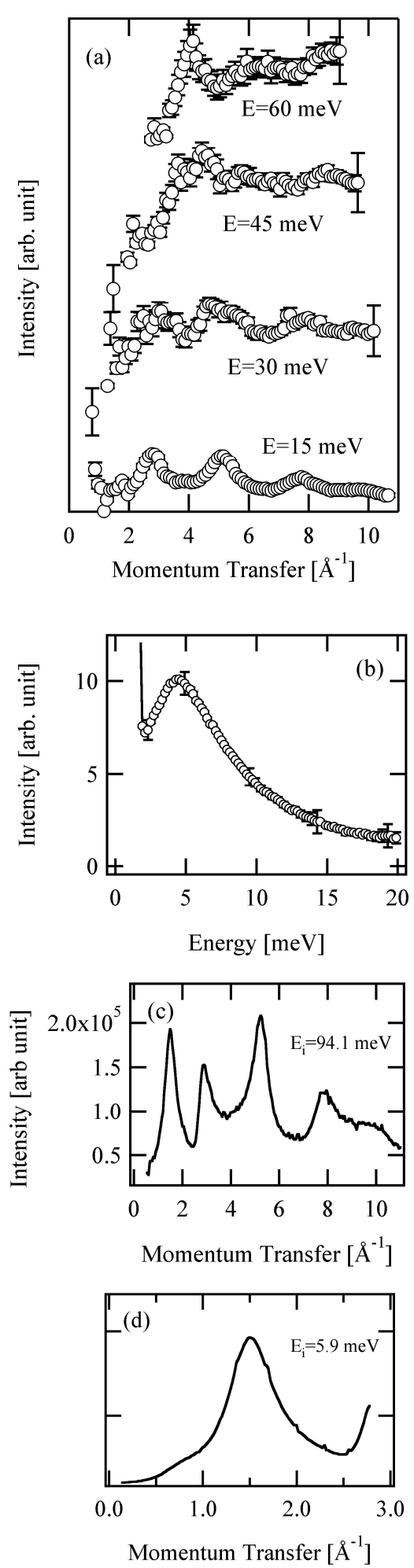

Fig. 4. Several plots extracted from the multiple $S(Q, E)$ maps in Fig. 3. (a) The constant- $E$ slices of the $S(Q, E)$ with $E_{i}=94.1 \mathrm{meV}$ for $E=15,30,45,60 \mathrm{meV}$. (b) The energy dependence of the $\mathrm{S}(\mathrm{Q}, \mathrm{E})$ with $E_{i}=23.6 \mathrm{meV}$ obtained by total integration along the $\mathrm{Q}$ direction. The so-called Boson Peak is observed around 5 $\mathrm{meV}$. The elastic parts of the $S(Q, E)$ with (c) $E_{i}=94.1 \mathrm{meV}$ and (d) $E_{i}=5.9 \mathrm{meV}$. The first sharp diffraction peak is observed around $1.5 \AA^{-1}$.
For the study of diffusion mechanism of mobile ions in solid state ionic materials, AMATERAS spectrometer at J-PARC also enables the QENS measurement with high resolution $(\Delta E<10 \mu \mathrm{eV})$ by the combination of multiple fast disk choppers [7]. The continuous $Q$ dependences of QENS spectra can be available at the AMATERAS through a two-dimensional $\mathrm{S}(\mathrm{Q}, \mathrm{E})$ map. Furthermore, AMATERAS was designed to facilitate the "multiEi QENS measurement" using multiple incident energies [6]. This experimental technique should be also greatly useful for the diffusion study of solid state ionic materials.

We would like to emphasize again that a wide variety of information can be extracted from only one data set of multi-Ei measurement. The highly efficient INS measurement by a new technique should enhance the research activities on the solid state ionic materials. The beam power of the J-PARC accelerator was $120 \mathrm{~kW}$ at the time we performed this study. Increasing the beam power to final goal (1 MW) is scheduled in accordance with the project plan. The combination of multi-Ei measurement and final beam power of $1 \mathrm{MW}$ at J-PARC will yield an unrivaled ability in various research fields of condensed matter physics.

\section{CONCLUSIONS}

In this paper, we have experimentally demonstrated that the multi-Ei measurement using a chopper spectrometer at the pulsed neutron source is markedly powerful for the dynamical study of a typical isotropic material, vitreous $\mathrm{SiO}_{2}$. The multiple two-dimensional $S(Q, E)$ maps are simultaneously obtained by one measurement. The full picture of dynamical properties, which include the overall feature over wide $Q-E$ space and the precise information in the low $Q-E$ region, is promptly captured. These results confirm that the multi-Ei measurement has the possibility to greatly advance the dynamical study of solid state ionic materials which are usually isotropic form (glassy or polycrystalline one).

\section{ACKNOWLEDGMENTS}

We are grateful to E. Kartini for the sample preparation of $(\mathrm{AgI})_{0.5}\left(\mathrm{AgPO}_{3}\right)_{0.5}$ and $\mathrm{AgPO}_{3}$ glasses. We are also grateful to the technical support team of J-PARC Center for their help in the experiment. This work was supported by a Grant-in-Aid for Specially Promoted Research (No. 17001001) from the Ministry of Education, Culture, Sports, Science and Technology of Japan, 
and a Grant-in-Aid for Scientific Research C (No. 20612016) from the Japan Society for the Promotion of Science.

\section{REFERENCES}

1. M. Nakamura, M. Arai, Y. Inamura and E. Kartini, J. Phys. Soc. Jpn. 79 (2010) Suppl. A 122.

2. E. Kartini, M. Nakamura, M. Arai, Y. Inamura, J. W. Taylor, and M. Russina, Solid State Ionics 180 (2009) 506.

3. M. Arai, H. Iwase, M. Nakamura, T. Otomo, E. Kartini, K. Itoh, S.J. Levett, and S.M. Bennington, AIP Conf. Proc. 832 (2006) 299.

4. M. Nakamura, M. Arai, E. Kartini, J. W. Taylor, and M. Russina, AIP Conf. Proc. 832 (2006) 504.

5. M. Nakamura, R. Kajimoto, Y. Inamura, F. Mizuno, M. Fujita, T. Yokoo and M. Arai, J. Phys. Soc. Jpn. 78 (2009) 093002.

6. M. Nakamura, K. Nakajima, R. Kajimoto, and M. Arai, J. Neutron Res. 15 (2007) 31.

7. K. Nakajima, M. Nakamura, R. Kajimoto, T. Osakabe, K. Kakurai, M. Matsuda, N. Metoki, S. Wakimoto, T. J. Sato, S. Itoh, M. Arai, K. Yoshida, and K. Niita, J. Neutron Res. 15 (2007) 13.
8. S. Satoh, S. Muto, N. Kaneko, T. Uchida, M. Tanaka, Y. Yasu, K. Nakayoshi, E. Inoue, H. Sendai, T. Nakatani, and T. Otomo, Nucl. Instrum. Methods Phys. Res., Sect. A 600 (2009) 103.

9. K. Nakayoshi, Y. Yasu, E. Inoue, H. Sendai, S. Satoh, S. Muto, N. Kaneko, T. Otomo, T. Nakatani and T. Uchida, Nucl. Instrum. Methods Phys. Res., Sect. A 600, (2009) 173.

10. T. Nakayama, Rep. Prog. Phys. 65 (2002) 1195.

11. M. Arai, Y. Inamura and T. Otomo, Philos. Mag. B 79 (1999) 1733.

12. M. Arai, Y. Inamura, T. Otomo, N. Kitamura, S. M. Bennington, and A. C. Hannon, Physica B 263-264 (1999) 268.

13. M. Nakamura, M. Arai, Y. Inamura, T. Otomo, and S. M. Bennington, Phys. Rev. B 66 (2002) 024203.

14. L. Börjesson, L. M. Torell, U. Dahlborg, and W. S. Howells, Phys. Rev. B 39 (1989) 3404.

15. E. Kartini, M. F. Collins, T. Priyanto, M. Yusuf, N. Indayaningsih, E. C. Svensson, and S. J. Kennedy, Phys. Rev. B 61 (2000) 1036. 